\title{
Rapid amygdala gamma oscillations in response to fearful facial expressions.
}

\section{$\operatorname{AUTHOR}(\mathrm{S})$ :}

Sato, Wataru; Kochiyama, Takanori; Uono, Shota; Matsuda, Kazumi; Usui, Keiko; Inoue, Yushi; Toichi, Motomi

\section{CITATION:}

Sato, Wataru ... [et al]. Rapid amygdala gamma oscillations in response to fearful facial expressions.. Neuropsychologia 2011, 49(4): 612-617

\section{ISSUE DATE:}

2011-03

URL:

http://hdl.handle.net/2433/138648

\section{RIGHT:}

C 2010 Elsevier Ltd; この論文は著者最終稿です。内容が印刷版と異な ることがありますので、引用の際には出版社版をご確認ご利用くださ い。 This is the Accepted Author Manuscript. Please cite only the published version. 


\section{Rapid amygdala gamma oscillations in response to fearful facial expressions}

Wataru Sato ${ }^{a}$, Takanori Kochiyama ${ }^{b}$, Shota Uono ${ }^{c}$, Kazumi Matsuda ${ }^{d}$, Keiko Usui ${ }^{\mathrm{d}}$, Yushi Inoue ${ }^{\mathrm{d}}$, and Motomi Toichi ${ }^{\mathrm{e}}$

\section{Institutional affiliation}

a The Hakubi Project, Primate Research Institute, Kyoto University, Inuyama, Aichi 484-8506, Japan.

${ }^{b}$ Brain Activity Imaging Center, Advanced Telecommunications Research Institute International, 2-2-2 Hikaridai, Seika-cho, Soraku-gun, Kyoto 619-0288, Japan.

c Department of Cognitive Psychology in Education, Kyoto University, Yoshida-honmachi, Sakyo-ku, Kyoto 606-8501, Japan.

d National Epilepsy Center, Shizuoka Institute of Epilepsy and Neurological Disorders, Urushiyama 886, Shizuoka 420-8688, Japan.

${ }^{\mathrm{e}}$ Faculty of Human Health Science, Graduate School of Medicine, Kyoto University, 53 Shogoin-Kawaharacho, Sakyo-ku, Kyoto 606-8507, Japan. 


\section{Abstract}

Neuroimaging studies have reported greater activation of the human amygdala in response to emotional facial expressions, especially for fear. However, little is known about how fast this activation occurs. We investigated this issue by recording the intracranial field potentials of the amygdala in subjects undergoing pre-neurosurgical assessment $(n=$ 6). The subjects observed fearful, happy, and neutral facial expressions. Time-frequency statistical parametric mapping analyses revealed that the amygdala showed greater gamma-band activity in response to fearful compared with neutral facial expressions at $50-150 \mathrm{~ms}$, with a peak at $135 \mathrm{~ms}$. These results indicate that the human amygdala is able to rapidly process fearful facial expressions.

\section{Keywords}

Amygdala; Emotional facial expression; Gamma oscillation; Intracranial field potential recording.

\section{Introduction}

Neuroimaging evidence indicates that the amygdala is critically involved in processing emotional facial expressions. Several studies have demonstrated that the human amygdala was more active in response to emotional than to neutral expressions, especially in the case of negative emotions such as fear (e.g., Breiter et al., 1996; Morris, Frith, Perrett, \& Rowland, 1996). Amygdala activity in response to emotional expressions has been shown to relate to emotional reactions to facial 
expressions (Sato, Yoshikawa, Kochiyama, \& Matsumura, 2004; Williams et al., 2001). This activation occurred automatically (Critchley et al., 2000), even in the absence of conscious awareness of faces (Whalen et al., 1998). These data suggest that the amygdala automatically conducts emotional processing of facial expressions.

Despite the accumulating evidence for amygdala activity in processing facial expressions, the temporal profile remains unclear. Some researchers (e.g., Adolphs, 2002; Morris, Ohman, \& Dolan, 1999) have speculated that amygdala activation may occur at an early stage of facial expression processing, because the amygdala receives input from subcortical as well as neocortical visual pathways (Jones \& Burton, 1976).

Some recent studies have investigated this issue by recording the magnetoencephalography (MEG) while participants were observing emotional and neutral facial expressions (Bayle, Henaff, \& Krolak-Salmon, 2009; Luo, Holroyd, Jones, Hendler, \& Blair, 2007; Luo et al., 2009; Hung et al., 2010; Maratos, Mogg, Bradley, Rippon, \& Senior, 2009). These studies consistently reported rapid amygdala activity changes in response to fearful or threatening, compared to neutral, expressions at approximately $100 \mathrm{~ms}$ following stimulus onset (i.e., $80-130 \mathrm{~ms}, 20-310$ $\mathrm{ms}, 40-270 \mathrm{~ms}, 100 \mathrm{~ms}$, and $50-250 \mathrm{~ms}$ ). Such data appear to support the hypothesis of rapid recruitment of amygdala activity in response to fearful expressions. However, the temporal profile observed across these studies was largely inconsistent, suggesting that the different estimation methods employed may have contributed to the discrepancies in the results. It should furthermore be emphasized that controversy as to whether the 
activity of such a deep, complex, brain structure as the amygdala, can be assessed using scalp MEG signals continues (Mikuni et al., 1997;

Papadelis, Poghosyan, Fenwick, \& Ioannides, 2009).

Intracranial field potential recordings in humans are able to provide direct evidence of neural activity with high temporal resolution. In this regard, a previous study investigated amygdala activity while subjects observed negative, positive, and neutral scenes by employing intracranial recording and time-frequency analyses (Oya, Kawasaki, Howard, \& Adolphs, 2002). This study reported higher gamma-band (around $40 \mathrm{~Hz}$ ) oscillations in the amygdala in response to negative scenes, as compared with both positive and neutral scenes, as early as $50-150 \mathrm{~ms}$ after stimulus onset. Such data suggest the possibility that the amygdala might show similar rapid gamma-band oscillations in response to fearful facial expressions, but this remains to be tested.

To depict the temporal, as well as frequency, profiles of electrical activity in the human amygdala in response to fearful facial expressions, we recorded the intracranial field potentials from the amygdala of six subjects who underwent pre-neurosurgical assessment. Subjects were shown fearful, happy, and neutral facial expressions using a dummy gender discrimination task. The amygdala field potential data were analyzed using time-frequency statistical parametric mapping (SPM) (Kilner, Kiebel, \& Friston, 2005). Based on the aforementioned intracranial recording and MEG data, we predicted that the amygdala would show higher gamma-band activity in response to fearful expressions than to neutral expressions as early as $50-150 \mathrm{~ms}$ following stimulus onset. 


\section{Methods}

\subsection{Subjects}

Six patients ( 5 females and 1 male; mean $\pm S D$ age, $34.5 \pm 7.9$ years) participated in the experiment. All subjects suffered from pharmacologically intractable focal epilepsy and received implantation of intracranial electrodes during a presurgical evaluation.

Electrophysiological and surgical evaluations suggested that the main epileptic foci were in the hippocampus for five subjects and in the lateral temporal cortex for one subject. The experiment was conducted $2.0-2.8$ weeks after electrode implantation. Neuropsychological assessments confirmed that language ability and everyday memory for all subjects were intact. The intelligence quotient (IQ), measured using the revised Wechsler Adult Intelligence Scale (WAIS-R), was in the normal range in five subjects, and in the mildly mentally retarded range in one subject (mean \pm $S D$ full-scale IQ: $91.8 \pm 19.2 ;$ mean $\pm S D$ verbal IQ: $86.7 \pm 12.0$; mean $\pm S D$ performance IQ: $100.7 \pm 27.3)$. During the experiment, no seizure activity was observed, and all subjects were mentally stable. All subjects were right handed, as assessed with the Edinburgh Handedness Inventory (Oldfield, 1971). All the subject had normal or corrected-to-normal visual acuity. Each subject gave written informed consent after the procedure was fully explained. The study was approved by the local institutional ethics committee.

\subsection{Anatomical MRI assessment}

Pre- and post-implantation anatomical assessments were conducted 
using structural magnetic resonance imaging (MRI) on a 1.5-T scanning system (Signa Twin Speed, General Electric Yokokawa) that employed T-1 weighted images. Three-dimensional fast-spoiled gradient-recalled acquisition was used with the following parameters: repetition time $=12$ $\mathrm{ms}$, echo time $=5 \mathrm{~ms}$, flip angle $=20^{\circ}$, matrix size $=256 \times 256$, field of view $=22 \times 22 \mathrm{~cm}, 76$ slices, resulting in voxel dimensions of $0.8594 \mathrm{x}$ $0.8594 \times 2.0 \mathrm{~mm}$. Pre-implantation MRI assessments and surgical evaluations showed no structural abnormalities in the bilateral amygdala of any subject.

Implantation of intracranial electrodes was accomplished using a stereotactic method (Mihara \& Baba, 2001). The patients received the implantation of 84-114 electrodes, the positions of which were customized depending on individual epileptic conditions. Implantation sites were selected based solely on clinical criteria. Post-implantation anatomical MRI assessments confirmed that the electrodes were successfully inserted into the bilateral amygdalae of all the subjects (Fig. 1a; Supplementary Fig. S1). A probabilistic cytoarchitectonic map of the amygdala (Amunts et al., 2005; Eickhoff et al., 2005) was also referenced to validate our selections (Fig. 1b).

Fig. 1

\subsection{Stimuli}

The stimuli consisted of grayscale photographs of seven individuals' faces from a standard set (Ekman \& Friesen, 1976) depicting fearful, happy, and neutral expressions. The luminance did not vary 
across emotions (one-way analysis of variance (ANOVA), $p>0.10$ ).

\subsection{Procedure}

The presentation of stimuli was controlled using SuperLab Pro 2.0 (Cedrus) and implemented on a Windows computer (FSA600, Teknos). The stimuli were presented on a 19-inch CRT monitor (GDM-F400, Sony) with a refresh rate of $100 \mathrm{~Hz}$ and a resolution of $1024 \times 768$ pixels. The subjects' responses were recorded using a response box (RB-400, Cedrus).

Experiments were conducted individually in a quiet room. Subjects were comfortably seated with the head supported by a chin-and-forehead rest, $0.57 \mathrm{~m}$ from the monitor. In this position, the stimuli subtended a visual angle of $11.1^{\circ}$ vertically $x 7.6^{\circ}$ horizontally.

Each stimulus was presented four times, resulting in 84 trials (28 trials each for the fearful, happy, and neutral photographs) for each subject. The stimuli were presented in random order. In each trial, following the presentation of a cross as a fixation point for $500 \mathrm{~ms}$, the stimulus was presented for $1000 \mathrm{~ms}$ in central vision. Then, the response panel was presented until the subject had completed the response. The subjects were instructed to wait without giving any response until presentation of the response panel, and were requested not to blink until then. Intertrial intervals were randomly varied between 2000 to $5000 \mathrm{~ms}$. To avoid habituation and drowsiness, the subjects were allowed a short rest halfway through the trials. Before data collection, the subjects were familiarized with the procedure through participation in a block of 10 training trials. 
The subjects were instructed to specify the gender of the presented faces. The response panel randomly showed either "Male?" or "Female?" and subjects indicated their "Yes" or "No" responses by pressing a button with the left or right forefinger. The allocation of buttons was counterbalanced across subjects. This task ensured the continued attention of the subjects to the stimuli and did not involve any explicit recognition of emotional expressions. Post-hoc debriefing confirmed that the subjects remained unaware that the purpose of the experiment concerned investigating the emotional variables.

\subsection{Data recording}

Intracranial field potential recordings were conducted using depth (0.8 $\mathrm{mm}$ diameter; Unique Medical) and subdural (2.3 $\mathrm{mm}$ diameter; Ad-tech) platinum electrodes. All electrodes were referenced to the electrodes embedded in the scalp of the midline dorsal frontal region. Impedances were balanced and maintained below $5 \mathrm{k} \Omega$. The data were amplified, filtered online (band pass: 0.5-120 Hz; notch: $60 \mathrm{~Hz}$ ), and sampled at $1000 \mathrm{~Hz}$ using an electroencephalograph (EEG) system (EEG-1100, Nihon Kohden). Horizontal and vertical electrooculograms (EOGs) were simultaneously recorded using Ag/AgCl electrodes (Nihon Kohden). Off-line visual inspection confirmed that no contamination of the EOGs on the intracranial field potentials had occurred. Correlation analyses for raw waveforms also showed no significant relationships between amygdala activity and either horizontal or vertical EOGs (mean $\pm S D r=-0.04 \pm 0.08,-0.07 \pm 0.09,-0.03 \pm 0.05$, and $-0.04 \pm 0.10$ for the left amygdala-horizontal EOG, left amygdala-vertical EOG, right 
amygdala-horizontal EOG, and right amygdala-vertical EOG, respectively; one-sample $t$-test for differences from zero after Fisher's $r$-to- $z$ transformation, $p s>0.10)$. These results correspond to those from a previous study, which also reported that intracranial recording was immune to eye-movement artifacts (Lachaux, Rudrauf, \& Kahane, 2003). Procedures were unobtrusively recorded using a video camera attached to the EEG. Off-line checks of the videotapes confirmed that all the subjects were engaged throughout the tasks.

\subsection{Data analysis}

We analyzed the electrodes implanted in the amygdalae. Intracranial field potential data were re-sampled using Psychophysiological Analysis Software 3.3 (Computational Neuroscience Laboratory of the Salk Institute), implemented using MATLAB 6.5 (Mathworks). The data were sampled for $1500 \mathrm{~ms}$ in each trial; pre-stimulus baseline data were collected for $500 \mathrm{~ms}$ (while the fixation point was presented), and experimental data were collected for $1000 \mathrm{~ms}$ after stimulus onset at a sampling rate of $200 \mathrm{~Hz}$. Any epoch that indicated an amplitude $>5 S D$ from the mean for each electrode for each subject was rejected as an artifact. The frequencies of the artifact-contaminated epochs for the amygdala electrodes were very low (1.2\%) and showed no significant systematic differences among the experimental conditions (one-way ANOVA, $p>$ $0.10)$.

Time-frequency SPM analyses (Kilner et al., 2005) were performed using SPM5 (http://www.fil.ion.ucl.ac.uk/spm/), implemented using MATLAB 6.5 (Mathworks). First, time-frequency (power) maps were 
calculated for each trial using continuous wavelet decomposition with seven-cycle Morlet wavelets from 4 to $60 \mathrm{~Hz}$. Average time-frequency maps were created for display purposes. The data were log-transformed to satisfy Gaussian assumptions for the subsequent analyses. Then, to ensure overlap across data and to enhance Gaussianity, the time-frequency maps were smoothed with a 2D Gaussian kernel of full width at half-maximum of $12 \mathrm{~Hz}$ in the frequency domain and $96 \mathrm{~ms}$ in the time domain (cf. Kilner et al., 2005).

The time-frequency maps were then entered into the general linear model (GLM) based on a fixed-effects analysis of the pooled error from all trials for every subject. The GLM included emotion (fear, happiness, neutral) and laterality (left, right) as factors of interest, and subject blocks (six subjects) as a factor of no interest. We analyzed the main effects of emotion (fearful versus neutral, fearful versus happy, and happy versus neutral) and the interactions between emotion and laterality using a one-dimensional linear contrast.

To ensure the assumption of independent and identically distributed error in GLM, correction for non-sphericity was applied. We modeled the 108 covariance components representing dependency and uneven variance between levels. The covariance components were estimated from the pooled active voxels (exceeding an uncorrected $F$ threshold of $p<0.001$ for any effects) with the restricted maximum likelihood procedure (Friston et al., 2002). Finally, the time-frequency $\operatorname{SPM}\{T\}$ was calculated for each contrast.

Statistical inference for the time-frequency $\operatorname{SPM}\{T\}$ was based on 
random field theory (Kilner et al., 2005). For the window of interest (WOI), we used the restricted time-frequency window (corresponding to the small volume correction in a standard SPM). Based on the data from a previous intracranial recording study (Oya et al., 2002), a time period of 50-150 ms following stimulus presentation was defined as the time region of the WOI. This time region covered common activity in the previous MEG studies for emotional faces (Bayle et al., 2009; Luo et al., 2007; Luo et al., 2009; Hung et al., 2010; Maratos et al., 2009).

According to the intracranial field potential data (Oya et al., 2002), the gamma-band range $(30-60 \mathrm{~Hz}$; cf. Karaka et al., 2001) was defined as the frequency region of the WOI. Other time-frequency ranges outside the WOI were corrected for whole time-frequency regions (-500-1000 ms, 4-60 Hz). Significant active time-frequency clusters were identified at a threshold of $p<0.05$ corrected for multiple comparisons within the small volume, with a height threshold of $p<0.01$ (uncorrected). The equivalent $Z$-value was used to report the results of the inference. As there was little evidence regarding the Gaussianity of the time-frequency random field (however, see Kilner et al., 2005), to validate the cluster size inference, we conducted a correction of the non-stationary smoothness using the VBM5.1 Toolbox (http://dbm.neuro.uni-jena.de/vbm/), which was based on the method employed by Hayasaka, Phan, Liberzon, Worsley, and Nichols (2004).

To determine the consistency of the effects across the subjects, we also conducted a conjunction analysis based on a global null hypothesis (Friston, Holmes, \& Worsley, 1999). In this analysis, we created a further 
GLM that allowed us to assess the interaction effect for the subjects. For the purpose of this analysis, we used a height threshold of $p<0.01$ (uncorrected).

For display purposes, effect size data of amygdala activity were extracted from the time-frequency maps. The data were sampled using the rectangular window, which extended to $30 \mathrm{~ms}$ in the time dimension and $6 \mathrm{~Hz}$ in the frequency dimension. The central coordinate was specified by the contrast between fearful and neutral expressions.

\section{Results}

Performance on the dummy gender-discrimination task was close to perfect $($ correct identification rate $=97.1 \%$; mean $\pm S D$ reaction time $=$ $771.2 \pm 153.3 \mathrm{~ms})$. No significant differences were found across emotions with regard to the number of correct responses or reaction times (one-way ANOVA, $p>0.10)$.

The time-frequency SPM for the comparison of fearful versus neutral expressions revealed a significant gamma-band activity between 50 and $150 \mathrm{~ms}$ (peak at $135 \mathrm{~ms}, 38 \mathrm{~Hz} ; Z=2.65$; cluster size, corrected $p<0.05$; Fig. 2a-c; Supplementary Fig. S2). A small activation was also observed in the gamma-band at 50-150 $\mathrm{ms}$ for fearful versus happy expressions, which failed to reach significance in the extent threshold (peak at $150 \mathrm{~ms}, 36 \mathrm{~Hz}$; $Z=2.68 ;$ cluster size, corrected $p=0.11)$. Conjunction analyses confirmed activation in the cluster of fearful versus neutral and fearful versus happy expressions $(p<0.01)$, indicating that amygdala activity was significant in each and every subject. No other significant main effects of emotion and 
no significant interactions between emotion and laterality were found in the WOI, which was also the case for all other time-frequency regions.

Fig. 2

To further confirm the rapid activation of the amygdala, we carried out a separate analysis for the amygdala in each hemisphere by using the WOI for the comparison of fearful versus neutral expressions. For the right amygdala, significant activation was found at $105 \mathrm{~ms}, 52 \mathrm{~Hz}$. In the case of the left amygdala, although no activation was found for the height threshold at the uncorrected $p<0.01$ level, activation was found using more liberal thresholds at the uncorrected level of $p<0.05$ at $140 \mathrm{~ms}, 37$ $\mathrm{Hz}$.

\section{Discussion}

The gamma-band activity of the amygdala was more pronounced in response to fearful compared with neutral facial expressions at 50-150 ms, with a peak at $135 \mathrm{~ms}$. A non-significant trend of gamma-band activation was also found in response to fearful versus happy expressions in this time range. Because subjects were engaged in a dummy task, amygdala activity can be regarded as primarily reflecting the automatic processes involved in responding to facial expressions. The automatic activity of the amygdala in response to fearful expressions confirms the results of previous neuroimaging studies (e.g., Breiter et al., 1996), although its temporal profile remained unknown. The present results also correspond to a previous intracranial field potential recording study that reported rapid 
amygdala activation in response to emotionally negative scenes (Oya et al., 2002). However, intracranial data of rapid amygdala activity to emotional faces have not been reported. Some previous scalp MEG studies have reported that rapid amygdala activity occurred in response to fearful expressions (e.g., Bayle et al., 2009), however, the results were inconsistent across these studies. Moreover, the estimation method employed in these studies remains controversial (e.g., Papadelis et al., 2009). To our knowledge, the current study is the first to provide direct evidence that the amygdala is involved in the rapid processing of fearful facial expressions.

What, then, are the implications of rapid amygdala activity at 50-150 ms for understanding emotional expression processing? In this respect, several previous scalp (e.g., Bentin, Allison, Puce, Perez, \& McCarthy, 1996) and subdurally recorded event-related-potential (ERP) studies (e.g., Allison, Puce, Spencer, \& McCarthy, 1999) reported that the first face-specific visual analysis in the visual cortices occurred at approximately 150-200 ms (for a review, see Rossion \& Jacques, 2008). A previous study that recorded intracranial field potentials and conducted time-frequency analyses also reported that face-specific activity, including broad frequency ranges from theta to gamma bands, occurred in the visual cortices at about $200 \mathrm{~ms}$ (Klopp, Marinkovic, Chauvel, Nenov, \& Halgren, 2000). When taken together with such findings, our data suggest that the processing of fearful facial expressions in the amygdala is faster than the first visual analysis of faces that takes place in the neocortex.

Several previous neuroimaging studies have indicated that amygdala 
activity in response to negative facial expressions was related to the activity of subcortical visual structures, such as the pulvinar and superior colliculus (e.g., Morris, de Bonis, \& Dolan, 2002). An earlier study also revealed that the amygdala was more active in response to fearful facial expressions, even in the presence of lesion to the bilateral primary visual cortices (Pegna, Khateb, Lazeyras, \& Seghier, 2005). Consistent with these findings, anatomical studies in monkeys have suggested that the amygdala receives input from the pulvinar (Jones \& Burton, 1976). Physiological and lesion studies in rats using auditory stimuli also provide evidence to support the involvement of the subcortical pathways to the amygdala in emotional processing (LeDoux, 1996). Taken together, our results suggest that the human amygdala processes fearful facial expressions at 50-150 ms via subcortical visual pathways and is able to bypass neocortical visual areas.

We found amygdala activity related to the processing of fearful expressions in the gamma band. This result agrees with previous investigations of intracranial recordings of amygdala activity for emotional scenes (Oya et al., 2002). This result is also consistent with previous intracranial data indicating that the amygdala produces high-frequency bursts, which include gamma-band activity, during spontaneous emotional behavior (Heath \& Gallant, 1964). Previous MEG studies in humans (Luo et al., 2007; Luo et al., 2009) and electrophysiological studies in animals (Bauer, Paz, \& Pare, 2007; Popescu, Popa, \& Pare, 2009) also suggest the existence of a relationship between emotional processing and gamma-band activity in the amygdala. Some 
cortical EEG studies in humans found that gamma-band activity was elicited in response to faces (Balconi \& Lucchiari, 2008; Balconi \& Pozzoli, 2009) and scenes (Keil et al., 2001; Muller, Keil, Gruber, \& Elbert, 1999) with emotional significance. Collectively, these data suggest that gamma-band activity in the amygdala plays an important role in emotional processing.

Our analysis of the amygdala in each hemisphere showed that the peak latency of the right amygdala was shorter than that of the left amygdala, although interaction related to laterality did not reach significance. This result is in line with previous neuroimaging studies indicating that the right, as compared with the left, amygdala showed dynamic changing patterns in response to facial expressions (Phillips et al., 2001; Wright et al., 2001). Other neuroimaging studies have shown that rapidly presented facial expression stimuli that did not reach conscious awareness activated the right, but not the left, amygdala (Morris et al., 1999; Nomura et al., 2004). Our data suggest that these hemodynamic activation patterns may reflect the rapid activation of the right, as compared with the left, amygdala for emotional facial expressions at 50-150 ms.

In contrast to our finding, an earlier study that employed intracranial recordings and ERP analysis reported no automatic amygdala activity in response to fearful facial expressions (Krolak-Salmon, Henaff, Vighetto, Bertrand, \& Mauguiere, 2004). Whereas ERP analysis can only detect low-frequency components in the time-frequency domain (Edwards et al., 2009), the time-frequency analysis employed in the present study allowed us to detect high- as well as low-frequency activity (Makeig, Debener, 
Onton, \& Delorme, 2004). Therefore, the discrepant results regarding automatic amygdala activity in response to fearful expressions may be explained by differences in the methodologies used.

Several previous behavioral studies indicated that emotional reactions to facial expressions can occur automatically (e.g., Johnsen, Thayer, \& Hugdahl, 1995). In addition, other studies suggested that these responses might be triggered rapidly, even before the conscious recognition of faces occurs (e.g., Murphy \& Zajonc, 1993). We suggest that the gamma oscillations found in the amygdala at 50-150 $\mathrm{ms}$ may be one of the neural correlates of such automatic, preconscious processing for fearful facial expressions.

Finally, a limitation of this study should be acknowledged. In this study, we used a simple facial gender discrimination task. It is possible that different tasks might modulate the rapid gamma-band activity and/or induce the activity at different time-frequency regions in the amygdala. Some neuroimaging studies have shown that intentional control of attention could suppress amygdala activity in response to emotional facial expressions (Bishop, Jenkins, \& Lawrence, 2007; Hsu \& Pessoa, 2007; Pessoa, McKenna, Gutierrez, \& Ungerleider, 2002; Pessoa, Padmala, \& Morland, 2005). Other studies found that verbal recognition for emotional facial expressions suppressed (Critchley et al., 2000; Hariri, Bookheimer, \& Mazziotta, 2000; Lange et al., 2003) or enhanced (Gorno-Tempini et al., 2001 ) amygdala activity. In future studies, participants should be asked to engage in such intentional cognitive processes in response to emotional facial expressions with the aim of investigating the time-frequency 
profiles of amygdala activity under those circumstances.

\section{Acknowledgments}

This study was supported by funds from the Benesse Corporation.

\section{References}

Adolphs, R. (2002). Neural systems for recognizing emotion. Current Opinion in Neurobiology, 12, 169-177.

Allison, T., Puce, A., Spencer, D. D., \& McCarthy, G. (1999). Electrophysiological studies of human face perception. I: Potentials generated in occipitotemporal cortex by face and non-face stimuli. Cerebral Cortex, 9, 415-430.

Amunts, K., Kedo, O., Kindler, M., Pieperhoff, P., Mohlberg, H., Shah, N. J., Habel, U., Schneider, F., \& Zilles, K. (2005). Cytoarchitectonic mapping of the human amygdala, hippocampal region and entorhinal cortex: Intersubject variability and probability maps. Anatomy and Embryology, 210,343-352.

Balconi, M., \& Lucchiari, C. (2008). Consciousness and arousal effects on emotional face processing as revealed by brain oscillations: A gamma band analysis. International Journal of Psychophysiology, $67,41-46$.

Balconi, M., \& Pozzoli, U. (2009). Arousal effect on emotional face comprehension: frequency band changes in different time intervals. Physiology \& Behavior, 97, 129-150.

Bauer, E. P., Paz, R., \& Pare, D. (2007). Gamma oscillations coordinate 
amygdalo-rhinal interactions during learning. The Journal of Neuroscience, 27, 9369-9379.

Bayle, D. J., Henaff, M. A., \& Krolak-Salmon, P. (2009). Unconsciously perceived fear in peripheral vision alerts the limbic system: A MEG study. PLoS One, 4, e 8207.

Bentin, S., Allison, T., Puce, A., Perez, E., \& McCarthy, G. (1996). Electrophysiological studies of face perception in humans. Journal of Cognitive Neuroscience, 8, 551-565.

Bishop, S. J., Jenkins, R., \& Lawrence, A. D. (2007). Neural processing of fearful faces: effects of anxiety are gated by perceptual capacity limitations. Cerebral Cortex, 17, 1595-1603.

Breiter, H. C., Etcoff, N. L., Whalen, P. J., Kennedy, W. A., Rauch, S. L., Buckner, R. L., Strauss, M. M., Hyman, S. E., \& Rosen, B. R. (1996). Response and habituation of the human amygdala during visual processing of facial expression. Neuron, 17, 875-887.

Critchley, H., Daly, E., Phillips, M., Brammer, M., Bullmore, E., Williams, S., Van Amelsvoort, T., Robertson, D., David, A., \& Murphy, D. (2000). Explicit and implicit neural mechanisms for processing of social information from facial expressions: A functional magnetic resonance imaging study. Human Brain Mapping, 9, 93-105.

Edwards, E., Soltani, M., Kim, W., Dalal, S. S., Nagarajan, S. S., Berger, M. S., \& Knight, R. T. (2009). Comparison of time-frequency responses and the event-related potential to auditory speech stimuli in human cortex. Journal of Neurophysiology, 102, 
$377-386$.

Eickhoff, S. B., Stephan, K. E., Mohlberg, H., Grefkes, C., Fink, G. R., Amunts, K., \& Zilles, K. (2005). A new SPM toolbox for combining probabilistic cytoarchitectonic maps and functional imaging data. Neuroimage, 25, 1325-1335.

Ekman, P., \& Friesen, W. V. (1976). Pictures of facial affect. Palo Alto, CA: Consulting Psychologist.

Friston, K. J., Glaser, D. E., Henson, R. N., Kiebel, S., Phillips, C., \& Ashburner, J. (2002). Classical and Bayesian inference in neuroimaging: Applications. Neuroimage, 16, 484-512.

Friston, K. J., Holmes, A. P., \& Worsley, K. J. (1999). How many subjects constitute a study? Neuroimage, 10,1-5.

Gorno-Tempini, M. L., Pradelli, S., Serafini, M., Pagnoni, G., Baraldi, P., Porro, C., Nicoletti, R., Umita, C., \& Nichelli, P. (2001). Explicit and incidental facial expression processing: An fMRI study. Neuroimage, 14, 465-473.

Hariri, A. R., Bookheimer, S. Y., Mazziotta, J. C. (2000). Modulating emotional responses: Effects of a neocortical network on the limbic system. Neuroreport, 11, 43-48.

Hayasaka, S., Phan, K. L., Liberzon, I., Worsley, K. J., \& Nichols, T. E. (2004). Nonstationary cluster-size inference with random field and permutation methods. Neuroimage, 22, 676-687.

Heath, R. G., \& Gallant, D. M. (1964). Activity of the human brain during emotional thought. In R. G. Heath (ed.), The role of pleasure in behavior: A symposium by 22 authors (pp. 83-106). New York: 
Harper \& Row.

Hsu, S. M., \& Pessoa, L. (2007). Dissociable effects of bottom-up and top-down factors on the processing of unattended fearful faces. Neuropsychologia, 45, 3075-3086.

Hung, Y., Smith, M. L., Bayle, D. J., Mills, T., Cheyne, D., \& Taylor, M. J. (2010). Unattended emotional faces elicit early lateralized amygdala-frontal and fusiform activations. Neuroimage, 50, $727-733$.

Johnsen, B. H., Thayer, J. F., \& Hugdahl, K. (1995). Affective judgment of the Ekman faces: A dimensional approach. Journal of Psychophysiology, 9, 193-202.

Jones, E. G., \& Burton, H. (1976). A projection from the medial pulvinar to the amygdala in primates. Brain Research, 104, 142-147.

Karaka, S., Baar-Eroglu, C., Ozesmi, C., Kafadar, H., \& Erzengin, O. U. (2001). Gamma response of the brain: A multifunctional oscillation that represents bottom-up with top-down processing. Int. J. Psychophysiol., 39, 137-150.

Keil, A., Muller, M. M., Gruber, T., Wienbruch, C., Stolarova, M., \& Elbert, T. (2001). Effects of emotional arousal in the cerebral hemispheres: A study of oscillatory brain activity and event-related potentials. Clinical Neurophysiology, 112, $2057-2068$.

Kilner, J. M., Kiebel, S. J., \& Friston, K. J. (2005). Applications of random field theory to electrophysiology. Neuroscience Letters, $374,174-178$. 
Klopp, J., Marinkovic, K., Chauvel, P., Nenov, V., \& Halgren, E. (2000). Early widespread cortical distribution of coherent fusiform face selective activity. Human Brain Mapping, 11, 286-293.

Krolak-Salmon, P., Henaff, M. A., Vighetto, A., Bertrand, O., \& Mauguiere, F. (2004). Early amygdala reaction to fear spreading in occipital, temporal, and frontal cortex: A depth electrode ERP study in human. Neuron, 42, 665-676.

Lachaux, J. P., Rudrauf, D., \& Kahane, P. (2003). Intracranial EEG and human brain mapping. Journal of physiology, Paris, 97, 613-628. Lange, K., Williams, L. M., Young, A. W., Bullmore, E. T., Brammer, M. J., Williams, S. C., Gray, J. A., \& Phillips, M. L. (2003). Task instructions modulate neural responses to fearful facial expressions. Biological Psychiatry, 53, 226-232.

LeDoux, J. E. (1996). The emotional brain: The mysterious underpinnings of emotional life. New York: Simon \& Schuster.

Luo, Q., Holroyd, T., Jones, M., Hendler, T., \& Blair, J. (2007). Neural dynamics for facial threat processing as revealed by gamma band synchronization using MEG. Neuroimage, 34, 839-847.

Luo, Q., Mitchell, D., Cheng, X., Mondillo, K., Mccaffrey, D., Holroyd, T., Carver, F., Coppola, R., \& Blair, J. (2009). Visual awareness, emotion, and gamma band synchronization. Cerebral Cortex, 19, $1896-1904$.

Makeig, S., Debener, S., Onton, J., \& Delorme, A. (2004). Mining event-related brain dynamics. Trends in Cognitive Sciences, 8, $204-210$. 
Maratos, F. A., Mogg, K., Bradley, B. P., Rippon, G., \& Senior, C. (2009). Coarse threat images reveal theta oscillations in the amygdala: A magnetoencephalography study. Cognitive, Affective, and Behavioral Neuroscience, 9, 133-143.

Mihara, T., \& Baba, K. (2001). Combined use of subdural and depth electrodes. In H. O. Luders, \& Y. G. Comair (eds.), Epilepsy surgery (2nd ed.) (pp. 613-621). Philadelphia: Lippincott Williams \& Wilkins.

Mikuni, N., Nagamine, T., Ikeda, A., Terada, K., Taki, W., Kimura, J., Kikuchi, H., \& Shibasaki, H. (1997). Simultaneous recording of epileptiform discharges by MEG and subdural electrodes in temporal lobe epilepsy. Neuroimage, 5, 298-306.

Morris, J. S., de Bonis, M., \& Dolan, R J. (2002). Human amygdala responses to fearful eyes. Neuroimage, 17, 214-222.

Morris, J. S., Frith, C. D., Perrett, D. I., \& Rowland, D. (1996). A differential neural response in the human amygdala to fearful and happy facial expressions. Nature, 383, 812-815.

Morris, J. S., Ohman, A., \& Dolan, R. J. (1999). A subcortical pathway to the right amygdala mediating "unseen" fear. Proceedings of the National Academy of Sciences of the United States of America, $96,1680-1685$.

Muller, M. M., Keil, A., Gruber, T., \& Elbert, T. (1999). Processing of affective pictures modulates right-hemispheric gamma band EEG activity. Clinical Neurophysiology, 110, 1913-1920.

Murphy, S. T., \& Zajonc, R. B. (1993). Affect, cognition, and awareness: 
Neuropsychologia 24

Affective priming with optimal and suboptimal stimulus exposures. Journal of Personality and Social Psychology, 64, $723-739$.

Nomura, M., Ohira, H., Haneda, K., Iidaka, T., Sadato, N., Okada, T., \& Yonekura, Y. (2004). Functional association of the amygdala and ventral prefrontal cortex during cognitive evaluation of facial expressions primed by masked angry faces: an event-related fMRI study. Neuroimage, 21, 352-363.

Oldfield, R. C. (1971). The assessment and analysis of handedness: The Edinburgh inventory. Neuropsychologia, 9, 97-113.

Oya, H., Kawasaki, H., Howard, M. A. 3, \& Adolphs, R. (2002). Electrophysiological responses in the human amygdala discriminate emotion categories of complex visual stimuli. Journal of Neuroscience, 22, 9502-9512.

Papadelis, C., Poghosyan, V., Fenwick, P. B., \& Ioannides, A. A. (2009). MEG's ability to localise accurately weak transient neural sources. Clinical Neurophysiology, 120, 1958-1970.

Pegna, A. J., Khateb, A., Lazeyras, F., \& Seghier, M. L. (2005). Discriminating emotional faces without primary visual cortices involves the right amygdala. Nature Neuroscience, $8,24-25$.

Pessoa, L., McKenna, M., Gutierrez, E., \& Ungerleider, L. G. (2002). Neural processing of emotional faces requires attention. Proceedings of the National Academy of Sciences of the United States of America, 99, 11458-11463. 
Pessoa, L., Padmala, S., \& Morland, T. (2005). Fate of unattended fearful faces in the amygdala is determined by both attentional resources and cognitive modulation. Neuroimage, 28, 249-255.

Phillips, M. L., Medford, N., Young, A. W., Williams, L., Williams, S. C., Bullmore, E. T., Gray, J. A., \& Brammer, M. J. (2001). Time courses of left and right amygdalar responses to fearful facial expressions. Human Brain Mapping, 12, 193-202.

Popescu, A. T., Popa, D., \& Pare, D. (2009). Coherent gamma oscillations couple the amygdala and striatum during learning. Nature Neuroscience, 12,801-807.

Rossion, B., \& Jacques, C. (2008). Does physical interstimulus variance account for early electrophysiological face sensitive responses in the human brain? Ten lessons on the N170. Neuroimage, 39, 1959-1979.

Sato, W., Yoshikawa, S., Kochiyama, T., \& Matsumura, M. 2004. The amygdala processes the emotional significance of facial expressions: An fMRI investigation using the interaction between expression and face direction. Neuroimage, 22, 1006-1013.

Whalen, P. J., Rauch, S. L., Etcoff, N. L., McInerney, S. C., Lee, M. B., \& Jenike, M. A. (1998). Masked presentations of emotional facial expressions modulate amygdala activity without explicit knowledge. Journal of Neuroscience, 18, 411-418.

Williams, L. M., Phillips, M. L., Brammer, M. J., Skerrett, D., Lagopoulos, J., Rennie, C., Bahramali, H., Olivieri, G., David, A. S., Peduto, A., \& Gordon, E. (2001). Arousal dissociates 
Neuropsychologia 26

amygdala and hippocampal fear responses: Evidence from simultaneous fMRI and skin conductance recording. Neuroimage, $14,1070-1079$.

Wright, C. I., Fischer, H., Whalen, P. J., McInerney, S. C., Shin, L. M., \& Rauch, S. L. (2001). Differential prefrontal cortex and amygdala habituation to repeatedly presented emotional stimuli. Neuroreport, 12, 379-383. 
a)

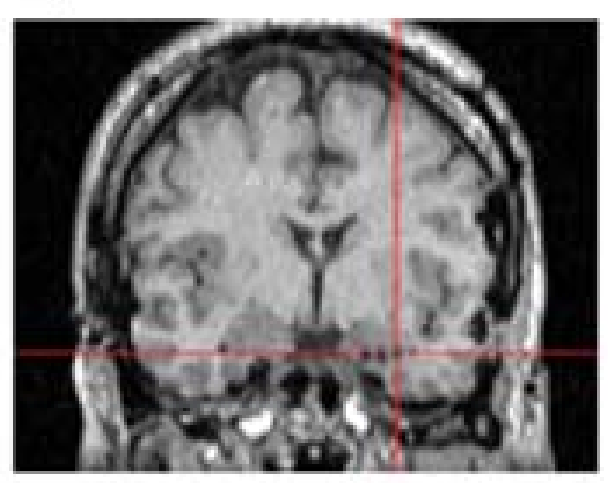

b)
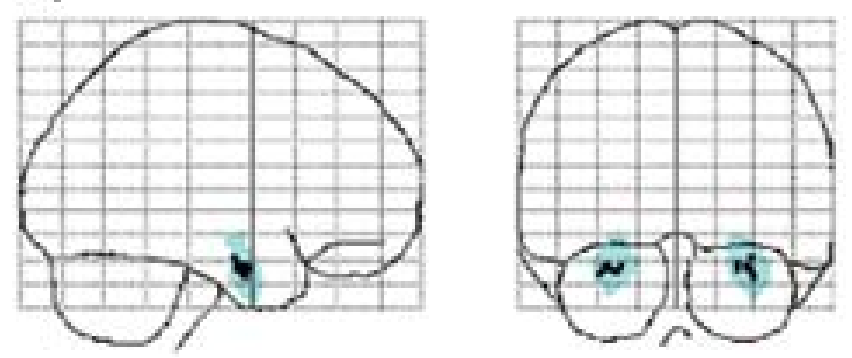

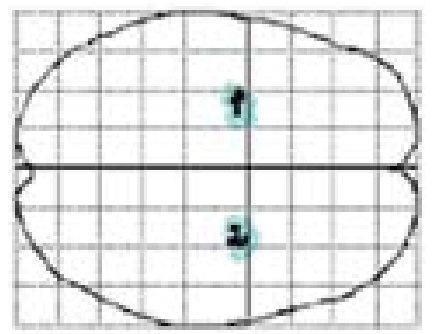

Fig. 1.

a) Representative anatomical magnetic resonance image. The red cross indicates the location of the amygdala electrode.

b) Glass brain projections of the coordinates of the amygdala electrodes (black points) in the Montreal Neurological Institute space. The anatomically defined location of the amygdala derived from the cytoarchitectonic map (Anatomy toolbox, Eickhoff et al., 2005) is represented in light blue. 
a)

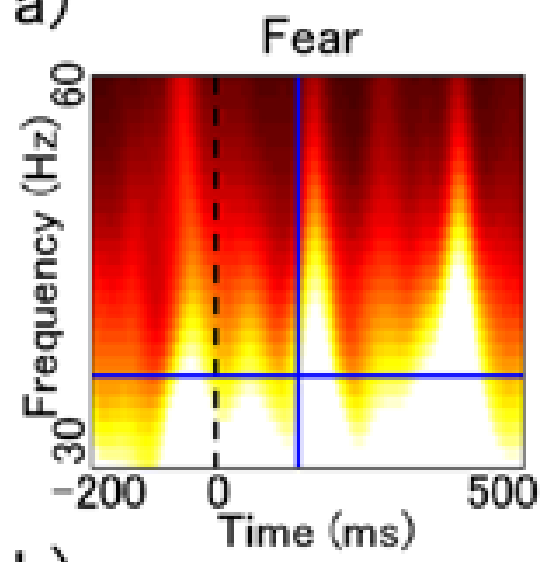

b)

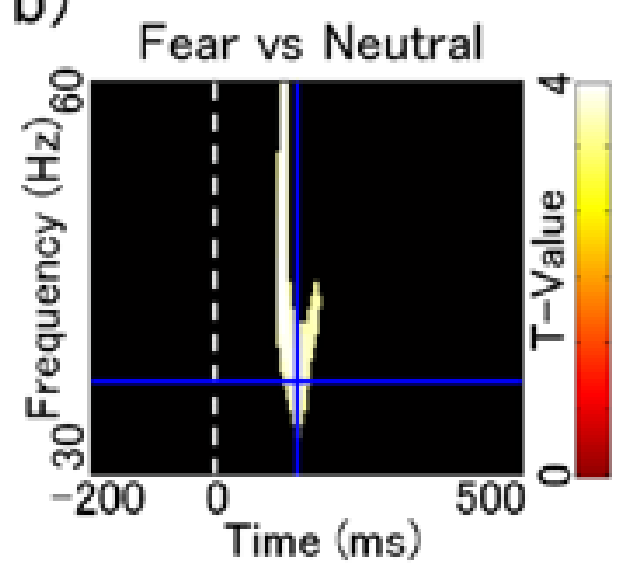

Happiness

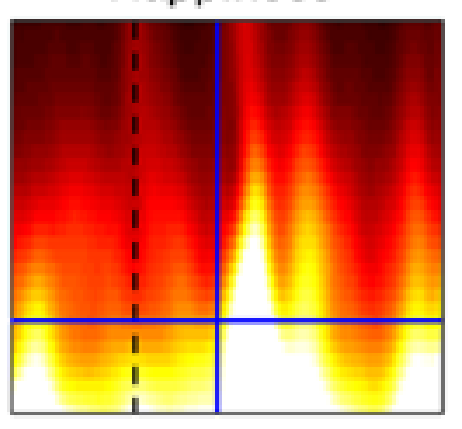

C)

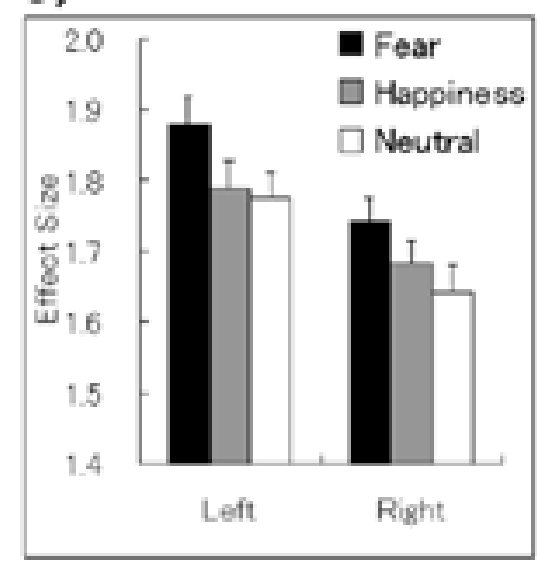

Fig. 2 .

a) Grand-average gamma-band time-frequency maps of the amygdala for fearful, happy, and neutral expressions. The results for both hemispheres are combined. The black dotted lines indicate stimulus onset. Blue crosses indicate the location of activation foci for fearful versus neutral expressions $(135 \mathrm{~ms}, 38 \mathrm{~Hz})$.

b) Statistical parametric maps for the gamma-band range that exhibited greater activation for fearful compared with neutral facial expressions $(p<$ 0.05, small volume corrected, with a height threshold of $p<0.01$, uncorrected). The white dotted lines indicate stimulus onset. The blue crosses indicate the location of activation foci for fearful versus neutral 
Neuropsychologia 29

expressions (135 ms, $38 \mathrm{~Hz})$.

c) Mean (with SE) effect size at the peak activation focus of the amygdala. 